\title{
Biopsy or cytology for diagnosing hepatic focal lesions?
}

\author{
Haeryoung Kim \\ Department of Pathology, Seoul National University Hospital, Seoul National University College of Medicine, Seoul, Korea
}

Keywords: Liver biopsy; Aspiration cytology; Liver neoplasms

\section{See Article on Page 305}

Pathological confirmation is an essential part of diagnosing neoplasms, as it is the only means of identifying the histological pattern (e.g., glandular, squamous) and it may also provide clues to identifying the cell lineage or origin, especially in situations where metastasis is a consideration. The two main methods of preoperative pathological examination are biopsies (e.g., core needle biopsy [CNB], incisional biopsy and punch biopsy) and cytopathology (e.g., fine needle aspiration cytology [FNAC], washing cytology, exfoliative cytology). For focal intrahepatic masses, the most commonly performed procedures are percutaneous CNB, transjugular liver biopsy and percutaneous FNAC. Recently, endoscopic ultrasound-guided fine needle aspiration biopsy is also receiving increasing attention. ${ }^{1,2}$

Although all these procedures potentially provide good quality tissue or cytology samples, they are invasive, and not without the risk of complications. In the setting of neoplasms, one rare but important complication of liver biopsy is needle tract seeding of the tumor cells, which has been reported in $\sim 3 \%$ overall for hepatocellular carcinomas (HCCs)..$^{3-5} \mathrm{~A}$ more common downside of needle biopsies is patient discomfort during the procedure, in ad- dition to the risk of bleeding and infection. FNAC is another wellestablished method for diagnosing focal lesions, which is less expensive compared to percutaneous $C N B$, and causes less discomfort to the patient, as it is usually performed with a narrower 22-gauge needle.

In this issue of Clinical and Molecular Hepatology, Huang et al. ${ }^{6}$ present the results of a direct comparison of the diagnostic performance of CNB, FNAC and touch imprint cytology (TIC) for diagnosing hepatic malignancies. In their analysis of 235 hepatic malignancies, the sensitivity of CNB (93.6\%) for diagnosing hepatic malignancies was superior to that of FNAC $(71.9 \%)$ and TIC (85.1\%) when all three procedures were performed, and adding FNAC to CNB only minimally increased the sensitivity of CNB. On the other hand, the authors found that FNAC alone provided excellent sensitivity (100\%) for diagnosing metastatic tumors that were hyperechoic on ultrasonography and located less than 4.5 $\mathrm{cm}$ from the needle insertion site. Based on their findings, the authors conclude that 1) CNB may be the most sensitive method for detecting hepatic malignancies as a whole, 2) the sensitivity of TIC approaches that of CNB for detecting metastatic lesions, and 3) FNAC is highly sensitive in the setting of superficially-located, hyperechoic and metastatic carcinomas.

Selection of the appropriate technique for hepatic neoplasm de-

\begin{abstract}
Abbreviations:
CNB, core needle biopsy; FNAC, fine needle aspiration cytology; HCC hepatocellular carcinoma; ROSE, rapid on-site evaluation; TIC, touch imprint cytology
\end{abstract}

\section{Corresponding author: Haeryoung Kim}

Department of Pathology, Seoul National University Hospital, Seoul National University College of Medicine, 103 Daehak-ro, Jongno-gu, Seoul 03080, Korea

Tel: +82-2-740-8322, Fax: +82-2-743-5530

E-mail: haeryoung.kim@snu.ac.kr

https://orcid.org/0000-0002-4205-9081 
tection should be based on the clinical context and the individual institutional experience and setting. In general, CNB provides more tissue material for histomorphological evaluation and ancillary studies. This is especially important in situations where the cytological features alone have limited value and evaluation of the tissue architecture provides essential clues for the diagnosis. For example, in the setting of small hepatic focal lesions where the differential diagnosis in question is well-differentiated hepatocellular neoplasm (e.g., HCC, hepatocellular adenoma) versus tumor-like lesion (e.g., focal nodular hyperplasia or regenerative nodule), it is necessary to evaluate features such as hepatic plate thickness, reticulin loss, presence of central fibrous scars, ductular reactions, changes in vascular profiles (e.g., sinusoidal capillarization, unpaired arteries, dystrophic blood vessels) and stromal invasion. The pattern of immunohistochemical expression - not merely its presence or absence - may also play a pivotal role in the diagnosis, for example, the map-like staining pattern of glutamine synthetase in focal nodular hyperplasia. Another setting where tissue evaluation is necessary is poorly differentiated malignant neoplasms. In these situations, sufficient tissue is often needed to perform panels of ancillary tests (such as immunohistochemistry, in situ hybridization and molecular sequencing) to characterize the cellular lineage and/or origin of the tumor.

However, FNAC also has its advantages. As it is usually performed with a narrower needle compared to CNB, it is useful for patients with poor liver function and risk of bleeding, for cases where the target nodules are located near the large vessels or when there is a need to penetrate the bowel, and for small and deeply-located lesions that require patient cooperation during the procedure.' In addition, in institutions where rapid on-site evaluation (ROSE) is available, FNAC may be used for assessment of adequacy during the procedure. Furthermore, it has also been demonstrated that tumor cellularity and tumor fraction were higher in FNACs compared to corresponding CNB tissues, suggesting that FNAC samples may be more suitable for molecular tests such as next-generation sequencing. ${ }^{8}$ Cell blocks may be prepared from FNAC material, on which immunostains can be performed. Liquidbased cytology may also have a role in FNAC of hepatic focal lesions, although further studies are needed. In institutions with experienced cytopathologists, hepatic FNAC has been found to be a highly sensitive and specific method of diagnosing hepatic malignancies. ${ }^{7,9}$

TIC of CNB specimens is performed in some institutions for onsite evaluation of adequacy (ROSE). ROSE, when available, provides real-time evaluation of the adequacy of the obtained tissue and therefore helps to avoid unnecessary additional needle passes. It should be noted that the TIC procedure itself may sometimes cause damage (e.g., crushing or drying artifacts) or depletion of the CNB tissue, potentially decreasing the diagnostic performance of $\mathrm{CNB} .^{10}$

Huang et al. ${ }^{6}$ demonstrated excellent sensitivity of FNAC for diagnosing metastatic lesions that were superficially located (less than $4.5 \mathrm{~cm}$ from the needle insertion site) and hyperechoic on ultrasound, suggesting a role for FNAC as the primary means of diagnosis in this clinical context, instead of CNBs. Although further validation would be required in independent and prospective cohort studies where the cytopathologists are blinded to the histopathology results and vice versa, considering the clinical diagnosis, the depth of the lesion and the echogenic features of a hepatic nodule may provide useful guides for selecting the appropriate procedure. However, it should be noted that these results are based on a categorical diagnosis of "positive for malignant cells" rather than a more specific diagnosis, such as the histological subtype and the site of origin etc. If FNAC is performed without a concurrent CNB, the amount of information yielded from the aspirate would depend on 1) the level of expertise of the cytopathologist, 2) the level of expertise of the radiologist and/or hepatologist performing the FNAC, 3) the presence of a multidisciplinary setting where the cytopathologist has access to detailed clinical and radiological information and 4) the availability of cell blocks for ancillary tests. Ancillary tests, such as immunocytochemistry, would be especially useful for the characterization of the primary site in the case of metastatic tumors.

\section{Conflicts of Interest}

The author has no conflicts to disclose.

\section{REFERENCES}

1. Chon HK, Yang HC, Choi KH, Kim TH. Endoscopic ultrasound-guided liver biopsy using a core needle for hepatic solid mass. Clin Endosc 2019;52:340-346.

2. Shah AR, Al-Hanayneh M, Chowdhry M, Bilal M, Singh S. Endoscopic ultrasound guided liver biopsy for parenchymal liver disease. World J Hepatol 2019;11:335-343.

3. Silva MA, Hegab B, Hyde C, Guo B, Buckels JA, Mirza DF. Needle track seeding following biopsy of liver lesions in the diagnosis of hepatocellular cancer: a systematic review and meta-analysis. Gut 2008:57:1592-1596. 


\section{CLINICAL and MOLECULAR}

4. Khalifa A, Rockey DC. The utility of liver biopsy in 2020. Curr Opin Gastroenterol 2020;36:184-191.

5. Rockey DC, Caldwell SH, Goodman ZD, Nelson RC, Smith AD; American Association for the Study of Liver Diseases. Liver biopsy. Hepatology 2009;49:1017-1044.

6. Huang SC, Liang JD, Hsu SJ, Hong TC, Yang HC, Kao JH. Direct comparison of biopsy techniques for hepatic malignancies. Clin Mol Hepatol 2021;27:305-312.

7. Wee A. Fine needle aspiration biopsy of the liver: algorithmic approach and current issues in the diagnosis of hepatocellular carcinoma. Cytojournal 2005;2:7.
8. Goldhoff PE, Vohra P, Kolli KP, Ljung BM. Fine-needle aspiration biopsy of liver lesions yields higher tumor fraction for molecular studies: a direct comparison with concurrent core needle biopsy. J Natl Compr Canc Netw 2019;17:1075-1081.

9. McHugh KE, Policarpio-Nicolas Md MLC, Reynolds JP. Fine-needle aspiration of the liver: a 10-year single institution retrospective review. Hum Pathol 2019;92:25-31.

10. Rekhtman N, Kazi S, Yao J, Dogan S, Yannes A, Lin O, et al. Depletion of core needle biopsy cellularity and DNA content as a result of vigorous touch preparations. Arch Pathol Lab Med 2015;139:907912. 